INPLASY

PROTOCOL

To cite: Zhang et al. Different exercise therapies for treating heart failure: A protocol for overview of systematic reviews and network Meta-analysis. Inplasy protocol 202080118. doi:

10.37766/inplasy2020.8.0118

Received: 28 August 2020

Published: 28 August 2020

Corresponding author:

Yingdong Li

287732149@qq.com

Author Affiliation:

Gansu University of Chinese Medicine

\section{Support: zyzx-2020-36}

Review Stage at time of this submission: Preliminary searches.

Conflicts of interest:

The authors declare that they have no competing interests.

\section{Different exercise therapies for treating heart failure: A protocol for overview of systematic reviews and network Meta-analysis}

\author{
Zhang, L1; Zhao, X2; Qiao, L3; Wei, W4; Wei, M5; Ding, J6; Li, Y7.
}

Review question / Objective: What are the efficacy and safety of different exercise therapies for the patients with heart failure?

Condition being studied: Patients with heart failure.

Information sources: The literature will search the following databases: PubMed, Web of Science, Cochrane Library, EMBASE, Chinese biomedical literature database (CBM), China National Knowledge Infrastructure(CNKI), Chongqing VIP (CQVIP) and Wan Fang database from inception to August 2020. Related words will be searched by medical subject headings (Mesh) or text word search. The retrieval strategy will be designed for each database. The language is limited to English and Chinese, and there are no other restrictions. The search strategy of PubMed and EMBASE will be shown. When the screening is completed, check the references list of included systematic reviews(SR) and Meta-analysis to determine whether other SR and Meta-analysis can be included.

INPLASY registration number: This protocol was registered with the International Platform of Registered Systematic Review and Meta-Analysis Protocols (INPLASY) on 28 August 2020 and was last updated on 28 August 2020 (registration number INPLASY202080118).

\section{INTRODUCTION}

Review question / Objective: What are the efficacy and safety of different exercise therapies for the patients with heart failure?

Condition being studied: Patients with heart failure.

\section{METHODS}

Search strategy: From the seven databases: PubMed, EMBASE.com, Web of Science, the Cochrane Library, Chinese biomedical literature database (CBM), Chinese National Knowledge Infrastructure (CNKI), Wan fang Database, Chongqing VIP (CQVIP) databases. to search for 
systematic or meta-analysis of different exercise therapies for Chronic heart failure. to identify relevant published and unpublished trials from systematic searches (The relevant systematic reviews, grey literature and guidelines will also be included). The search strategy will be adapted to each database, the search terms include " Heart Failure ","Coronary Artery Disease", "Exercise"," Exercise Therapy ", " systematic review ", " MetaAnalysis ", etc. The language is limited to English and Chinese, and there are no other restrictions.

Participant or population: According to World Health Organization and "Guidelines for the Diagnosis and Treatment of Heart Failure(HF) in China" ,this study will include participants who were diagnosed as HF, irrespective sex, age and duration.

Intervention: Any exercise therapies.

Comparator: Routine drug therapy.

Study designs to be included: Systematic review(SR), Meta-analysis and the eligible randomized controlled trial (RCTs) were selected from the included systematic reviews and updated RCTs from the above systematic reviews to August2020.

Eligibility criteria: Eligibility criteria for the review are based on the PICOS framework. We will include studies that meet the following criteria: (1) Patients diagnosed with Heart Failure; (2) The intervention group includes any exercise therapies; (3) The controlled group is any active drugs that being used in clinical practice; (4) Systematic review(SR), Meta-analysis and the eligible randomized controlled trial (RCTs) were selected from the included systematic reviews and updated RCTs from the above systematic reviews to August2020.

Information sources: The literature will search the following databases: PubMed, Web of Science, Cochrane Library, EMBASE, Chinese biomedical literature database (CBM), China National Knowledge Infrastructure(CNKI),
Chongqing VIP (CQVIP) and Wan Fang database from inception to August 2020.Related words will be searched by medical subject headings (Mesh) or text word search. The retrieval strategy will be designed for each database. The language is limited to English and Chinese, and there are no other restrictions. The search strategy of PubMed and EMBASE will be shown. When the screening is completed, check the references list of included systematic reviews(SR) and Meta-analysis to determine whether other SR and Metaanalysis can be included.

Main outcome(s): The primary outcomes were peak oxygen uptake, left ventricular ejection fraction(LVEF), left ventricular end-diastolic diameter (LVEDD), ventilation over carbon dioxide slope, oxygen uptake efficiency slope, exercise oscillatory ventilation, rest and peak pulmonary endtidal $\mathrm{CO} 2$.

Additional outcome(s): The Secondary outcomes were blood pressure, heart rate, 6-minute walk test (6MWT), brain natriuretic peptide (BNP) and quality of life(QoL).

Data management: Two researchers applied EndNote X 8.0 (Thomson Reuters (Scientific) LLC Philadelphia, PA, US) Software manages literature. The following information was extracted from the predesigned Microsoft Excel 2019 software table: author, year of publication, number of references, number of cases, interventions, control measures, method quality assessment tool, safety assessment, and risk of bias.

Quality assessment / Risk of bias analysis: Two independent reviewers will check titles/abstracts of all potential literatures, and will remove irrelevant studies. Through read full-text of potential trials to determine if they fulfill all inclusion criteria. Excluded studies will be listed with specific reasons in a table. Any divergences will be solved by consulting a third reviewer. The Assessment of Multiple Systematic Reviews-2 (AMSTAR-2) measurement tool 
was used for methodological evaluation. The (PRISMA) statement was used for reporting quality evaluation, it consists of 27 items. The GRADE tool was used to grade the evidence quality of the major outcome indicators.

Strategy of data synthesis: First, pairwise direct comparisons were performed, and then network meta-analysis was performed to analyze the two-arm studies in which the three-arm studies were divided into pairwise comparisons. For dichotomous variables, odds ratios (ODD Radio,OR) and $95 \%$ confidence intervals $(\mathrm{Cl})$ were used to evaluate the effectiveness of different exercise therapies in improving heart function. For continuous variables, they were expressed as standard mean difference (SMD) and $95 \% \mathrm{CI}$. The heterogeneity of pairwise comparison was determined by 12 and $P$ values. If $12 \leq 50 \%$ and $P \geq 0.05$, there was no significant heterogeneity in the included study. Significant heterogeneity was indicated when I $2>50 \%$ or $P<0.05$. we will use the random effect model of Stata.15.0 to conduct paired meta-analysis of direct evidence. By comparing direct and indirect estimates and calculating inconsistencies within each evidence closed-loop, differences between direct and indirect evidence for the same comparison are explored. The node-splitting method is used to evaluate whether there is inconsistency in all existing comparative analysis, that is, whether there is inconsistency in direct and indirect evidence. For the comparison of the effectiveness of intervention measures, the therapeutic effect of intervention measures was ranked by using the surface area under the cumulative curve and the average level as evaluation indexes. The larger the surface area under the cumulative curve and the smaller the average level were, the better the therapeutic effect of intervention measures would be. Comparative corrected funnel plots are used to detect potential small sample effects or publication bias.

Subgroup analysis: If possible, we will do some extra subgroup analyses according to the results of heterogeneity and inconsistency. We will also investigate the sources of heterogeneity to determine the robustness and reliability of the consolidated results.

Sensibility analysis: If possible, we will conduct a series of sensitivity analyses, trials where missing data have been imputed will be excluded, trials where high risk of bias rating have been assessed, and trials where only included patients comorbidity with other cardiovascular diseases will be excluded. We will also investigate the sources of heterogeneity to determine the robustness and reliability of the consolidated results.

Language: English.

\section{Country(ies) involved: China.}

Keywords: Heart failure; Exercise therapy; Systematic review; Meta-analysis.

\section{Contributions of each author:}

Author 1 - Li Zhang - Study design, data extraction, analyzed the data ,wrote the protocol and revised the manuscript.

Author 2 - Xiaopeng Zhao - Provided methodological advice, polished and revised the manuscript, provided expertise on treatments, outcomes and related knowledge.

Author 3 - Lijuan Qiao - Searched the literature, In charge of extracting data and verification.

Author 4 - Wanxia Wei - Searched the literature, and analyzed the data.

Author 5 - Min Wei - Searched the literature, In charge of extracting data and verification.

Author 6 - Jin Ding - was the corresponding author and approved the final version of the manuscript.

Author 7 - Yingdong Li - was the corresponding author, responsible for all work of the review. 